\title{
A NEW STRESS-FIELD BASED MODEL TO SIMULATE THE MULTIPLE CRACKING PROCESS IN ENGINEERED CEMENTITIOUS COMPOSITES (ECC)
}

\author{
CONG LU* , CHRISTOPHER K. Y. LEUNG ${ }^{\dagger}$ AND VICTOR C. LI ${ }^{\dagger \dagger}$ \\ *the Hong Kong University of Science and Technology \\ Hong Kong \\ e-mail: cluab@ust.hk \\ ${ }^{\dagger}$ the Hong Kong University of Science and Technology \\ Hong Kong \\ e-mail: ckleung@ust.hk \\ ${ }^{\dagger \dagger}$ University of Michigan \\ Michigan, USA \\ e-mail: vcli@umich.edu
}

Key words: ECC, Simulation, Stress field, Matrix strength distribution

\begin{abstract}
Engineered cementitious composites (ECC) are materials exhibiting strain-hardening behavior up to several percent tensile strain with the formation of multiple cracks. The conditions for achieving multiple cracking have been investigated in literature, but issues such as the sequential formation of multiple cracking, the number of cracks formed at a particular stress level (which governs material ductility) and the crack opening at a particular strain (which affects durability) are seldom addressed.

In this paper, a new model to simulate the overall stress-strain relation for an ECC member is developed. For a bridging fiber at a certain inclination angle, the fiber stress and the stress transferred to the matrix at various distances from the crack is derived with consideration of slip hardening behavior, snubbing effect at the fiber/matrix interface and fiber rupture. At any applied loading, the matrix stress at any distance from the crack can be obtained by summing up the stress transfer from all the acting fibers. With a stochastic approach to describe the continuous variation of matrix strength along the member, the stress field in the matrix is compared to the distributed matrix strength to determine the positions of new cracks. The strain at a particular stress level, as well as the corresponding number and openings of cracks, can then be obtained.

Using this model, the effects of various micro parameters on the ductility and strength of ECC member can be simulated. This provides guidelines for the design of ECC to achieve better mechanical performance.
\end{abstract}

\section{INTRODUCTION}

As the most widely used construction materials, the potential of cementitious composites such as concrete and mortar is greatly limited by their brittleness, which makes them vulnerable to cracking. Addition of fibers is an effective way to control the cracks. Through proper material design guided by micromechanics, engineered cementitious composites (ECC) with tensile strain capacity up to several percent strain accompanied by 
the formation of multiple fine cracks have been developed [1].

In the design of ECC, physical behaviour at three scale levels, for the fiber, a crack bridged by fibers, and a tensile member containing inherent cracks, should be studied. Micromechanics theories to model single fiber behavior have been developed since 1970s [24]. Based on the single fiber behavior, the crack behavior can also be computed by models proposed in [3] and [5-7]. However, there has been no effective model to link the single crack behaviour with the tensile behaviour of a member which is most closely related to real applications. The prerequisites to achieve multiple cracking have been investigated in the literature $[3,8]$, but only a few researches have studied the development of sequential multiple cracks on an ECC member, such as the number/openings of cracks at any given stress, which determine the ductility of ECC. In [9], an approximate minimum crack spacing was derived. However, without the consideration of nonuniform matrix strength, the model predicts the simultaneous formation of cracks with uniform spacing, which is inconsistent with reality. In [10], a stochastic model with distributed matrix strength and fiber content was proposed, which captures the influences of flaw-size determined matrix strength and fiber content. With the finite element method, another stochastic model in [11] studied the influence of sample size which affects inhomogenity of material properties. Both models in $[10,11]$ emphasized the influence of material randomness, but the stress transfer in the vicinity of a crack was overlooked, which essentially determines the saturation extent of multiple cracking. To be more detailed, the stress transfer situation will change along with increasing applied stress and occurrence of fiber rupture. In an newly developed model based on stress transfer considering the above factors, simulation was conducted on a tensile member [12] and the crack number/openings can be obtained at various stress/strain levels. However, in that model, slip hardening behavior, which is significant for PVA-ECC was not considered. Also, the strength variation in [10] was assumed to follow the Weibull distribution and the strength varies randomly along the member, implying two neighbouring sections can exhibit very different strength. This is inconsistent with test results showing the formation of cracks in one region followed by cracking in other regions, which can be explained by the fact that lower strength results from local increase in porosity. This is often due to poorer compaction which happens not just along one section but within a certain region of the specimen. There is hence a correlation between the strength of neighbouring sections. This correlation was also studied and discussed in $[10,11]$.

To address the issues stated above, a new model is developed to simulate the overall stress-strain relation for an ECC member. After the first crack is formed in a fiber composite, if the fibers crossing the cracked section are strong enough to carry additional loading, multiple cracking will occur [1]. The bridging fibers under high tension will transfer stress back to the surrounding matrix through the interaction at the fiber/matrix interface, thus enabling the matrix to reach its fracture strength again at a certain distance from the crack [3]. In this study, for a bridging fiber at a certain inclination angle, the fiber stress and the stress in matrix transferred from the fiber is derived in terms of distance from the crack [5], considering slip hardening behavior, snubbing effect at the fiber exit point and fiber rupture. The stress field in the matrix near a crack can then be calculated by summing up the transfer from all the bridging fibers. A stochastic method is adopted to consider the strength variation along the member, with correlation among neighbouring sections. By comparing the calculated stress field with distributed matrix strength, the positions of new cracks are determined. At any given stress, the number of cracks, the crack openings as well as the strain can all be obtained.

\section{STRESS NEAR A CRACK}

When a crack forms in the matrix, the stress released by the matrix is carried by the 
bridging fibers. At the cracked plane, the fibers are stretched to a high strain level while the cracked matrix relaxes to zero strain. The strain difference between fibers and matrix results in debonding and sliding along their interface through which the additional stress in fibers is transferred back to the matrix until the strain difference is eliminated. For a randomly orientated fiber, the stress transfer is realized by: 1) a pulley force at the exit point of an inclined fiber; 2) interfacial friction along the debonded length of fiber.

In this section, the stress transfer for a single fiber is studied first. Then the stress filed near a crack can be derived by summing up the contributions from every single fiber.

\subsection{Stress transfer for a single fiber}

\subsubsection{Debonding stage}

A schematic diagram is shown in Fig. 1 for a fiber perpendicular to crack with an embedment length of $l_{e}$, and debonded over a length $l_{d}$ along which fiber sliding occurs under frictional bond strength $\tau_{0}$. In the figure $l_{b}$ stands for the length of fiber that is still bonded to the matrix. $x$ is the distance from the crack plane, and the load in the fiber at the exit point is denoted as $P_{d}(x=0)$. With the frictional stress transfer along the debonded length, the fiber load at the end of the debonded zone, $P_{d}\left(x=l_{d}\right)$ is:

$$
P_{d}\left(x=l_{d}\right)=P_{d}(x=0)-\pi d_{f} l_{d} \tau_{0}
$$

where $d_{f}$ is the diameter of the fiber. The fiber strain at the end of the debonded zone is then:

$$
\varepsilon_{f}\left(x=l_{d}\right)=\frac{P_{d}\left(x=l_{d}\right)}{\left(\pi d_{f}^{2} / 4\right) E_{f}}=\frac{P_{d}(x=0)-\pi d_{f} l_{d} \tau_{0}}{\left(\pi d_{f}^{2} / 4\right) E_{f}}
$$

The corresponding matrix strain is:

$$
\varepsilon_{m}\left(x=l_{d}\right)=\frac{\pi d_{f} l_{d} \tau_{0}}{\left(\pi d_{f}^{2} / 4\right)\left(V_{m} / V_{f}\right) E_{m}}
$$

At the end of the debonded zone, the strain in fiber and matrix should be equal:

$$
\varepsilon_{f}\left(x=l_{d}\right)=\varepsilon_{m}\left(x=l_{d}\right)
$$

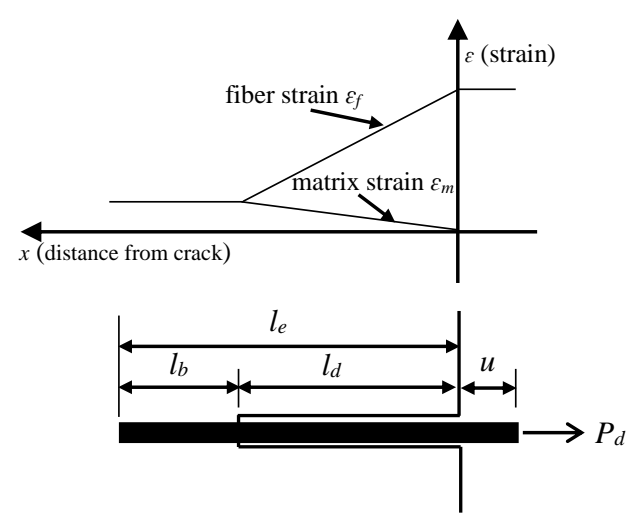

Figure 1: Fiber in debonding stage

The difference of elastic deformation between fiber and matrix over the debonded length results in the fiber displacement $u$ :

$$
u=\frac{P_{d}(x=0)-\frac{1}{2} \pi d_{f} l_{d} \tau_{0}}{\left(\pi d_{f}^{2} / 4\right) E_{f}} \times l_{d}-\frac{\frac{1}{2} \pi d_{f} l_{d} \tau_{0}}{\left(\pi d_{f}^{2} / 4\right)\left(V_{m} / V_{f}\right) E_{m}} \times l_{d}
$$

From Eqs. (2-5), the fiber load $P_{d}(x=0)$ and debonded length $l_{d}$ can be obtained in terms of $u$ :

$$
\begin{gathered}
P_{d}(x=0)=\pi \sqrt{\tau_{0} u E_{f} d_{f}^{3}(1+\eta) / 2}, \text { when } u<u_{0} \\
l_{d}=\sqrt{\frac{u E_{f} d_{f}}{2 \tau_{0}(1+\eta)}}
\end{gathered}
$$

where $u_{0}=2 \tau_{0} l_{e}^{2}(1+\eta) / E_{f} d_{f}$ represents the fiber displacement when the fiber is completely debonded.

\subsubsection{Pullout stage}

After the fiber is completely debonded from the matrix (as shown in Fig. 2), the pullout stage commences. Assuming the elastic deformation of fiber to be relatively small compared with the embedded length of fiber, the fiber length remaining in the tunnel $l_{p}$ can be expressed as:

$$
l_{p}=l_{e}-u+u_{0}
$$




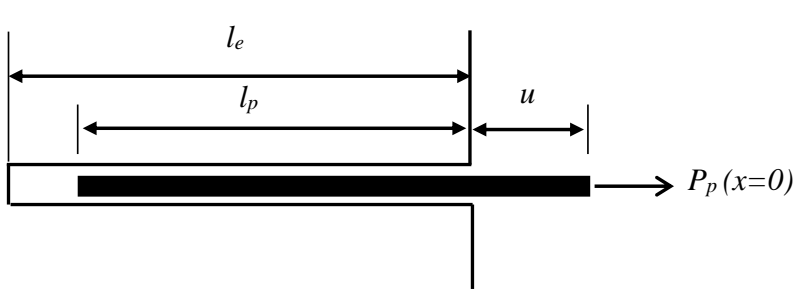

Figure 2: Fiber in pullout stage

For some types of fibers, particularly PVA fibers, slip-hardening behavior can be observed. In this analysis, the frictional bond strength in pullout stage is assumed to vary linearly with the slip distance following an slip-hardening coefficient $\beta$ [4]. Therefore the fiber load can be expressed as:

$$
P_{p}(x=0)=\pi \tau_{0}\left(l_{e}-u+u_{0}\right)\left(d_{f}+\beta\left(u-u_{0}\right)\right) \text {, when } u>u_{0}
$$

\subsubsection{Stress transferred by pulley force}

When a crack is formed and opened, an inclined fiber will exhibit a change in angle at the exit point (Fig.3). It is assumed by Aveston et al [2] that the inclined fiber behaves as a string bent over a frictionless pulley. The fiber forces on the two sides of the pulley are then the same. However, experimental results for real composite systems often show an increase of crack bridging force with fiber inclination, which can be explained by the 'snubbing' effect when the fiber (which is modelled as a string) is passing over a frictional pulley. Assuming Coulomb friction at the pulley, it can be shown that the bridging force $P(\theta)$ for a fiber at an inclining angle $\theta$ is amplified by a factor of $\exp (f \cdot \theta)$ (Fig. 3) relative to the fiber perpendicular to the crack, where $f$ is the snubbing coefficient to be determined experimentally.

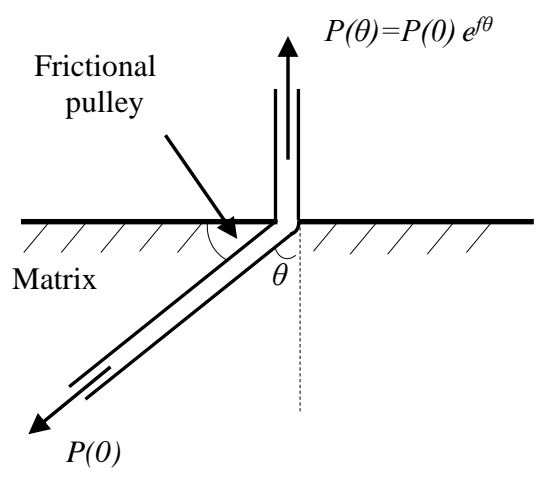

Figure 3: Schematic diagram for snubbing effect

The axial forces at both sides of a single fiber adjacent to the exit point are illustrated in Fig.4. Considering force equilibrium, the force transferred back to matrix along the crack opening direction by a single inclined fiber can be derived as:

$$
P_{\text {pulley }}=P(0) \mathrm{e}^{f \theta}-P(0) \cos \theta
$$

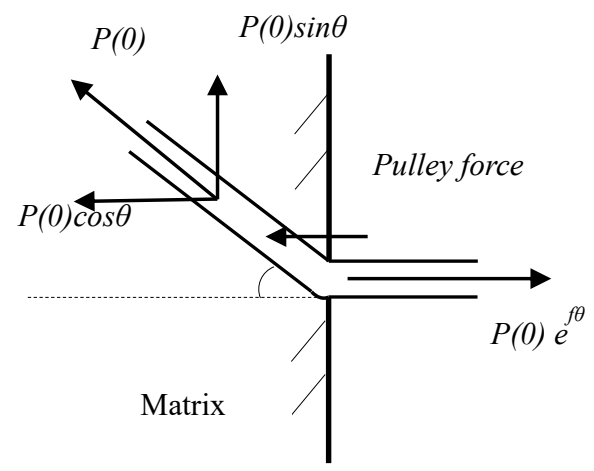

Figure 4: Force equilibrium at exit point

\subsubsection{Stress transferred by frictional force}

Away from the cracked plane, the additional stress taken by fibers will be transferred back to the surrounding matrix through the fiber/matrix interface. For a fiber in debonding stage, the stress can be transferred within the debonded length $l_{d}$ in Eq. (7) with a transfer rate of $\pi d_{f} \tau_{0}$, while for a fiber in pullout stage, the transfer is within the fiber length that is still in contact with the matrix, denoted as $l_{p}$ in Eq. (8), with a transfer rate of $\pi\left(d_{f}+\beta\left(u-u_{0}\right)\right) \tau_{0}$. 


\subsubsection{Fiber statuses considering two way pullout}

For fibers that do not exhibit slip hardening behavior, when the short embedment side of the fiber is completely debonded, the long embedment side will remain anchored and will never enter the pullout stage as the fiber force decreases with the pullout of short side. However, for the PVA fiber, slip hardening behavior results in the possibility of two way pullout, i.e., both sides could be pulled out simultaneously if the pullout load on the short side is increased to sufficiently high values due to interfacial slop hardening [5]. In this case, five different statuses of a fiber should be defined to better classify the stress transfer condition.

1) Two way debonding: when the stress is smaller than the stress at which the short side of fiber completes debonding, both sides are in debonding stage.

2) Pullout-debonding: when the short side of fiber is being pulled out while the stress has not exceeded the stress at which long side of fiber completes debonding, the long side is still in debonding stage.

3) Two way Pullout: when the stress in the short side being pulled out exceeds the stress at which long side of fiber completes debonding, both sides are in pullout stage.

4) Ruptured: when the stress exceeds the apparent strength of fiber, the fiber will undergo rupture.

5) Pulled out: when the short side of the fiber is completely pulled out from the matrix.

After classifying the fiber statuses, the corresponding stress transfer can be calculated according to whether that side is at the debonding or pullout stage. When the fiber is ruptured or completely pulled out, the fiber will lose its capacity to transfer any load.

\subsection{Stress transfer for a single crack}

The bridging stress and stress transfer can be calculated by summing up the contribution of every single fiber. One way is to integrate analytically [12] based on the probability distributions of fiber inclination and embedment length. However, the analytic method usually results in tedious mathematical equations and requires long computation time. A discrete method is therefore adopted and the fibers are divided into groups based on the combination of their inclination and embedment length. The number of fibers in each group can then be calculated based on the probability distributions of inclination and embedment length. For theoretically random distributed fibers in 3D space, the embedment length on the short side can be described by a uniform distribution from 0 to $l_{f} / 2$, and the probability density for inclination angle $\theta$ is $\sin \theta$ in the range of 0 to $\pi / 2$. In reality, the distribution of fiber orientation can be different from theoretical randomness, in which case the experimentally determined distribution can be adopted in the model. In this study, fibers at different inclination angle are equally divided into 100 groups from 0 to $\pi / 2$, while those with different embedment length are similarly divided into 100 groups from 0 to $l_{f} / 2$. A total of 10,000 different groups are hence considered. The bridging stress, transferred pulley stress and transferred frictional stress in each group are calculated and summed up to obtain the stresses resuelted from the opening of the crack.

One point to be noted in the calculation is that the embedment length distribution is only taken for the short embedment side from 0 to $l_{f}$ 2. The frictional transfer is normally different for the long side and short side. Thus when calculating the transferred frictional stress for a certain embedment length, the frictional stress from both the long embedment side and short embedment side are calculated and averaged.

\subsection{Results on stress field near a crack}

To calculate the stress field with the model derived above, parameters of matrix, fibers as well as the fiber/matrix interface are required. Based on references [4, 5, 7], the selected parameters for the simulation are listed in Table 1. 
Table 1: Example of the construction of one table

\begin{tabular}{cll}
\hline \multirow{4}{*}{ Fiber } & Fiber length $l_{f}(\mathrm{~mm})$ & 12 \\
& Fiber diameter $d_{f}(\mathrm{~mm})$ & 0.039 \\
& Fiber elastic modulus $E_{f}(\mathrm{GPa})$ & 22 \\
& Nominal fiber strength $\sigma_{f u}^{n}(\mathrm{MPa})$ & 1060 \\
\hline \multirow{5}{*}{ Matrix } & Elastic modulus $E_{m}(\mathrm{GPa})$ & 20 \\
\hline \multirow{3}{*}{ Interface } & Frictional bond $\tau_{0}(\mathrm{MPa})$ & 1.31 \\
& Snubbing coefficient $f$ & 0.2 \\
& Fiber strength reduction factor $f$ & 0.33 \\
& Slip Hardening Parameter $\beta$ & 0.58 \\
\hline
\end{tabular}

With the parameters in Table 1, the stresscrack opening relation is shown in Fig. 5 and the peak strength is slightly higher than $6 \mathrm{MPa}$ at roughly $130 \mu \mathrm{m}$ crack opening. Based on the stress transfer analysis described above, the stress field adjacent to a crack is derived and shown in Fig. 6 for various crack openings from $10 \mu \mathrm{m}$ to $130 \mu \mathrm{m}$. As can be seen, the stress due to the pulley force, which is the lowest value found at the crack location in each curve, increases with increasing crack opening until the peak strength at $130 \mu \mathrm{m}$ is reached. This is because the pulley stress is amplified by the increasing crack bridging stress.

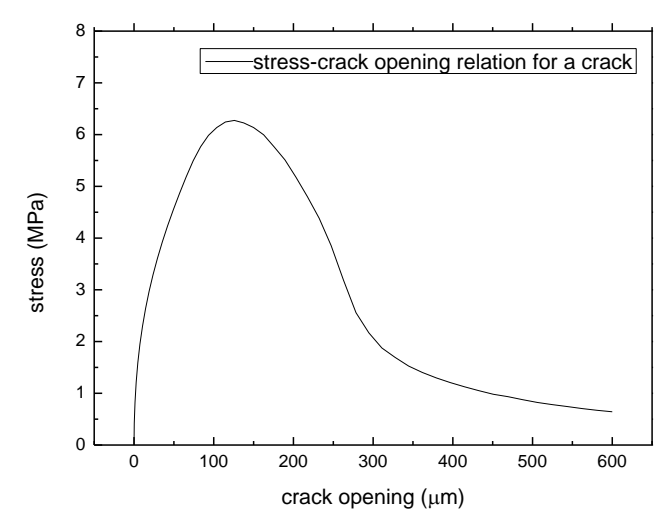

Figure 5: Stress-cracking opening relation for a crack

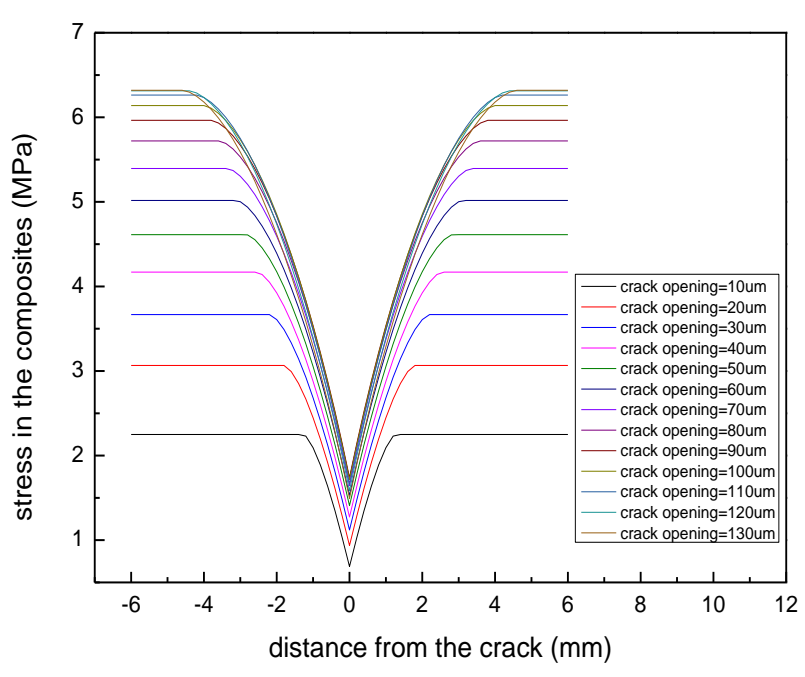

Figure 6: Stress field near a crack at various crack opening

Starting from the crack plane, the additional stress taken by the fibers is continuously transferred back to the matrix by interfacial friction until the matrix stress returns to the value before cracking at a certain distance. In the case illustrated in Fig. 6, this transfer distance increases with increasing crack opening. This can be explained by the fact that larger crack opening results in high fiber deformation and longer debonded length for longer embedded fibers. It therefore takes a longer distance for the frictional transfer to be completed. On the other hand, with higher stress, fiber rupture and pullout are getting more and more significant (as shown in Fig. 7) which reduce the efficiency of stress transfer.

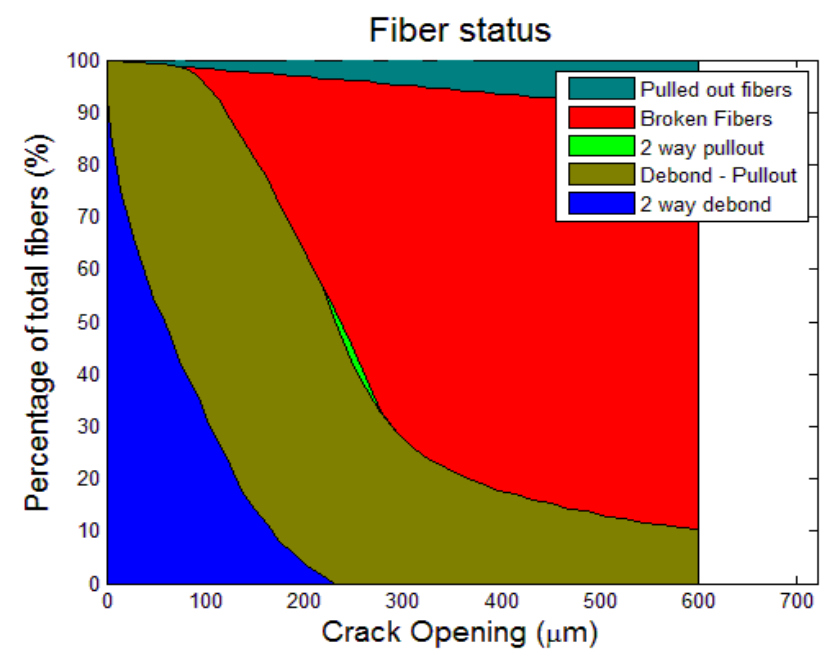

Figure 7: Percentage of fibers with different statuses 


\section{VARYING MATRIX STRENGTH}

The classical ACK model [2] assumes uniformly distributed matrix fracture strength in the tensile member. In this situation, when the matrix fracture strength has been reached, all cracks will occur simultaneously at a uniform spacing. This is inconsistent with reality where cracks occur in sequence and the crack spacing is highly random instead of being uniform. Such a phenomenon is caused by varying matrix strength at different sections. In [13], the non-uniform matrix strength resulting from varying flaw size was analyzed and a Weibull distribution for the matrix strength/flaw size was suggested, which is also adopted in [12]. For the Weibull distribution, the probability for members to have a certain largest flaw/weakest section is increasing exponentially with its length, which agrees with the real situation. However, the relation of strength between adjacent sections has not been considered. To illustrate this point, a Weibull distribution describing the matrix strength distribution is shown in Fig. 8. As can be seen, there is no correlation between the strength of neighboring sections so a section with very high strength can be right next to one with very low strength. However, according to experimental observation, cracking is often found to occur in a certain region first before other parts are cracked. In other words, the cracking strength is similar for neighboring sections within a weak region. This can be explained by the fact that low strength is caused by high porosity resulted from poor compaction or high local water/binder ratio, which occurs over a region of the specimen rather than at a single section. To properly describe the cracking process, a model with some correlation between the strength of adjacent sections is required.

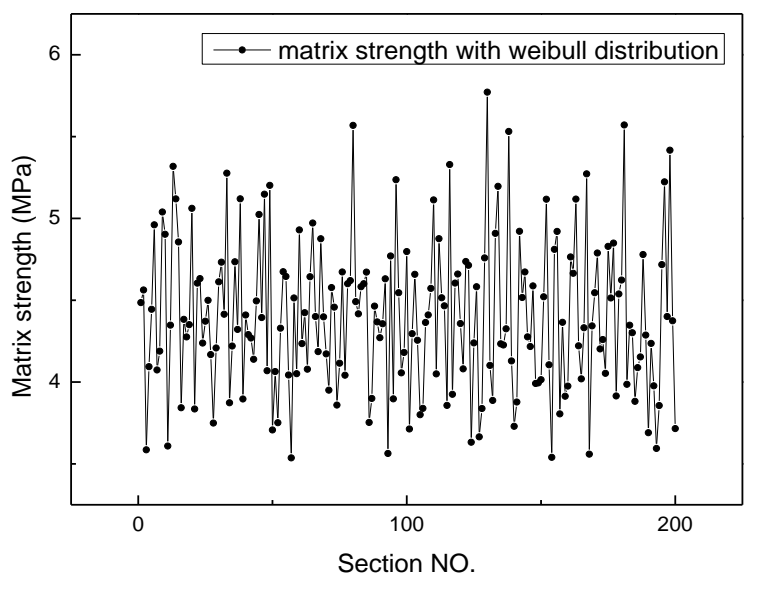

Figure 8: Matrix strength with weibull distribution

To describe this characteristic of matrix strength distribution, the study in [10] suggested to distribute the flaws with random location and size following normal distribution, which is also adopted in present study. The member is first divided into a number of sections. (In our case, the $40 \mathrm{~mm}$ long specimen is divided into 200 sections.) Then a number of flaws are assigned to the member and their centroid locations are taken to be uniformly distributed along the length Without loss of generality, flaws are assumed to be spheroidal in shape. Each flaw is then assigned a size based on normal distribution. As shown in Fig. 9, this will generate several distributed flaws with various locations and sizes in the member. Let $d_{\text {flaw }}(0)$ be the diameter of a certain flaw. For a section intersecting the pore at a longitudinal distance of $\mathrm{z}$ from the middle section (see Fig.9), the flaw diameter $d_{\text {flaw }}(z)$ can be easily calculated by:

$$
d_{\text {flaw }}(z)=2 \sqrt{d_{\text {flaw }}(0)^{2} / 4-z^{2}}
$$

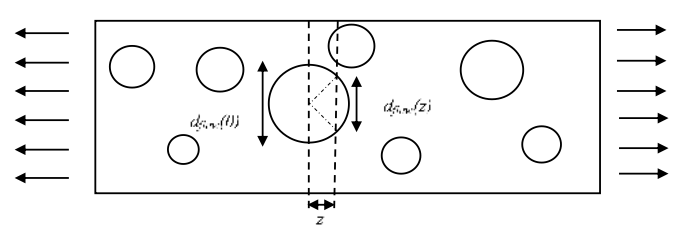

Figure 9: Schematic diagram for calculating flaw size

Based on Eq. (11), the sizes of flaws intersected by each section can be calculated and the fracture strength is taken to be governed by the largest flaw. In this manner, 
the dominating flaw size will vary continuously between adjacent sections as shown in Fig. 10.

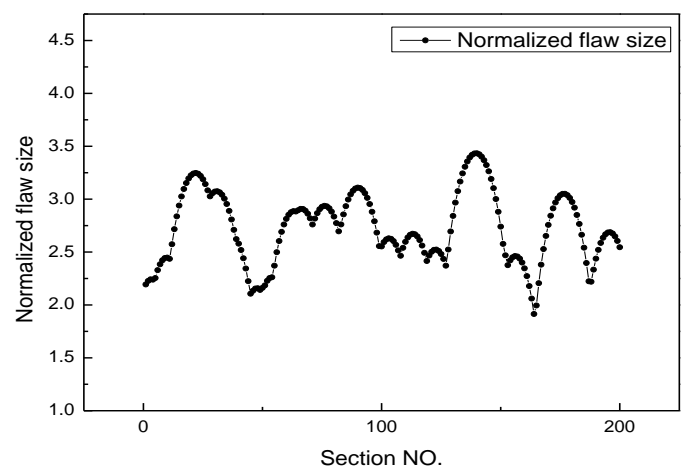

Figure 10: Normalized flaw size distribution along the member

Assuming the largest flaw on each section to act as a penny-shaped crack as in [3], the normalized first cracking strength of a crack with bridging fibers can be obtained from:

$\tilde{\sigma}_{f c}=\left(\frac{2}{4+f^{2}}\right)\left(1+\mathrm{e}^{\pi f / 2}\right)\left[\frac{\sqrt{\pi}}{2} \frac{\tilde{K}}{\tilde{c}}+\left(\frac{4}{3} \sqrt{\tilde{c}}-\frac{1}{2} \tilde{c}\right)\right]$

where the $\sigma_{f c}, K$ and $c$ with ( ) are quantities that have been normalized by certain values, and the details can be referred to [3]. The calculated matrix strength distribution based on the parameters in Table 2 is shown in Fig. 11, which varies continuously making it more representative of the real situation.

Table 2: parameters of matrix strength distribution

\begin{tabular}{llc}
\hline $\begin{array}{l}\text { Normalized } \\
\text { distribution } \\
\text { of flaw size }\end{array}$ & $\begin{array}{l}\text { Mean value } \\
\text { Standard derivation }\end{array}$ & 1.3 \\
\hline $\begin{array}{l}\text { Other } \\
\text { parameters }\end{array}$ & $\begin{array}{l}\text { Number of flaws } \\
\text { Normalized crack } \\
\text { tip toughness }\end{array}$ & $\begin{array}{c}100 \\
0.188\end{array}$ \\
\hline
\end{tabular}

It is clear that there is some inconsistency in the above approach - spherical pores are assumed in determining the flaw size in each section but the flaws are assumed to be pennyshaped. However, this approach can provide a better representation of real strength variation and give reasonable results for the stress vs strain curve and crack development (as shown in the next section). It can therefore be treated as an effective semi-empirical method to obtain a realistic strength distribution. In the above, simple assumptions have been made on the flaw shape as well as distributions of the flaw size and its centroid location. With relevant test results in the future, these can all be customized to give better predictions.

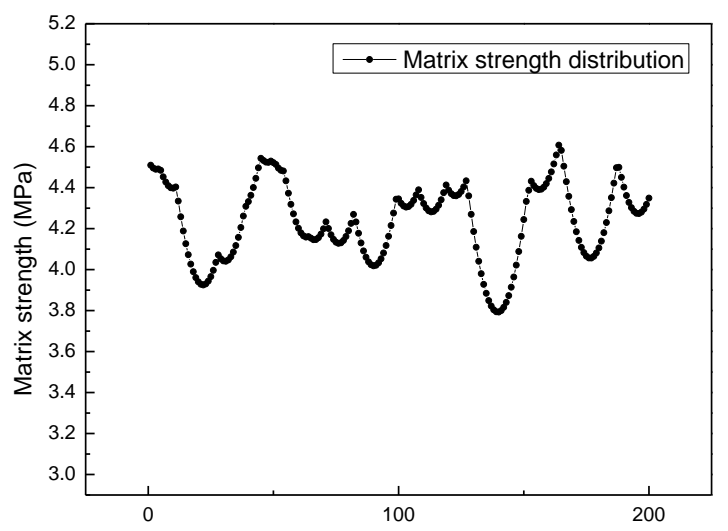

Figure 11: Matrix strength distribution

\section{SIMULATION OF MULTIPLE CRACKING PROCESS}

With the distributed matrix strength and calculated stress transfer near a crack, simulation of a tensile test on ECC can be performed. In the simulation, the stress field at each step is compared to the fracture strength distribution. If the strength is exceeded at any section, a new crack is formed. In the subsequent steps, the varying matrix stress field around this new crack is calculated from stress transfer.

As mentioned earlier, the $40 \mathrm{~mm}$ tensile member is divided into 200 sections (each section has a thickness of $0.2 \mathrm{~mm}$ ). Every section is assigned a ranking number from 1 to 200 that with the ranking number increases with strength of the section. Firstly, a stress equal to the strength of section 1 (weakest section) is applied. A crack opens up and the stress field near the section is modified by the stress transfer.

Then, at subsequent steps, the stress is increased to the strength of the section that ranks next, until the highest strength (for section 200) is reached. When the stress is increased to the strength of section i, the new stress field at this applied stress will be 
recalculated. Checking for cracking will be conducted, starting from the weakest uncracked section. If its strength has been reached by the stress filed, a new crack will open up and the stress field should be recalculated near this section. Otherwise, this section retains un-cracked status, and the section with the next lowest strength is checked. The flowchart for the simulation is demonstrated in Fig. 12.

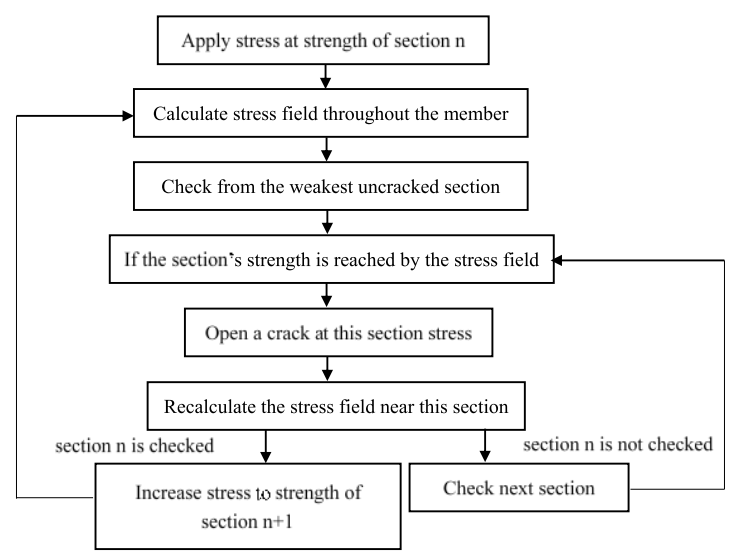

Figure 12: Flow chart to simulate multiple cracking

If the peak bridging stress of the fiber is lower than the strength of the strongest section, the above procedure will be ceased at the peak bridging stress. If the peak stress turns out to be higher, additional steps from the strength of the strongest section to the peak stress will be added, and the examination procedures are similar to previous steps.

\section{RESULTS AND DISCUSSION}

Following the simulation process, the number of cracks at each step can be obtained while the opening at each crack is directly calculated from the applied stress. The elongation at each step due to cracking is then computed as the sum of crack opening for all the cracks (the elastic deformation of material between the cracks is negligible compared with the crack opening). Dividing the total elongation by the specimen length, the composite strain is obtained. The simulated stress-strain curve is plotted in Fig. 13. To compare with simulation results, an experimental curve for a $35 \mathrm{~mm}$ ECC specimen from [14] is also shown in Fig. 13. From the plots, the strain hardening trend, first cracking strength and strain capacity of simulation and experiment are very close, while the peak strength from simulation is $1 \mathrm{MPa}$ higher. As many parameters are taken from the literature and not directly measured, the purpose of the comparison is not to prove that the model can perfectly predict the tensile behavior of ECC in real situation. However, the simulation is shown to provide satisfying prediction of real test results in many aspects.

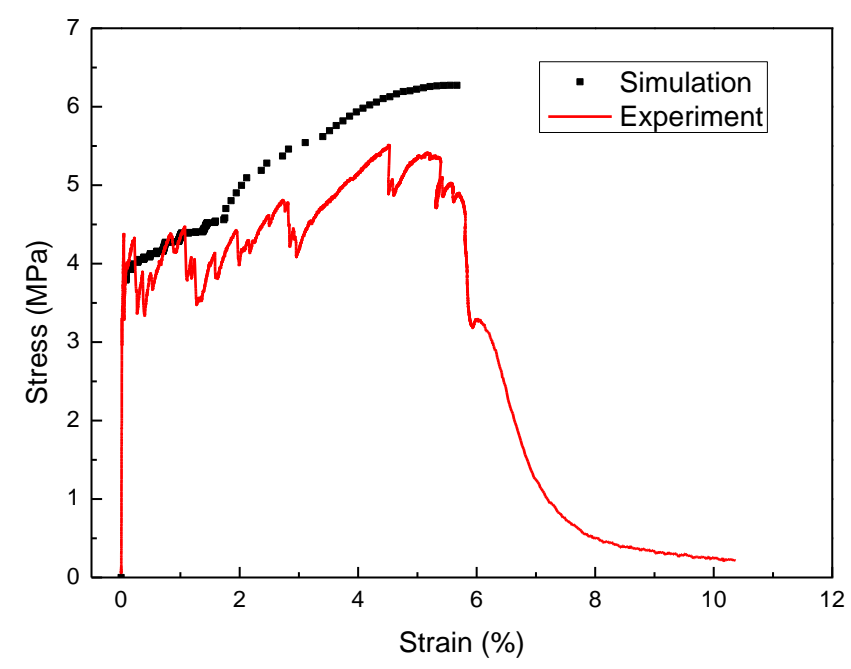

Figure 13: Comparison of stress-strain relation from simulation and experiment

What is more interesting, Fig. 14 shows the comparison between the simulated crack number to the result from an image processing technique proposed in [14] (for the same specimen in Fig. 13). Their similarity is remarkable, both showing rapid increase in crack number until steady state value of $0.46 / \mathrm{mm}$. This shows the capability of the model in simulating the multiple cracking process, which is important for durability design as the crack number and opening at a given strain (e.g., that under serviceability state) is of interest. 


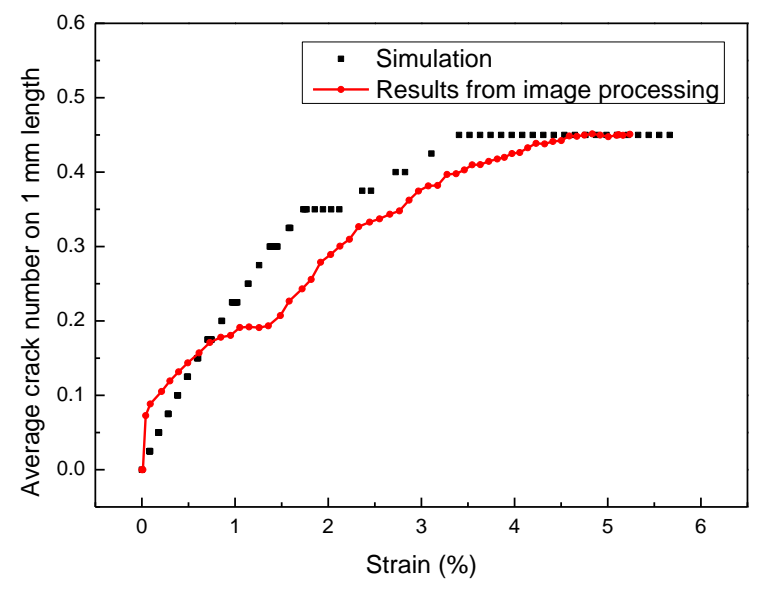

Figure 14: Comparison of crack number development from simulation and experiment

In Fig. 15, the distributed matrix strength vs stress field along the member is shown at four stages (first crack, 4.5 MPa, 5.2 MPa and peak strength). With increasing remote stress, the cracks are getting closer to saturation and stress transfer is taking part over a larger portion of the specimen. At the latter stage, every section of the matrix is having stress transfer from the fibers and no section can truly reach the applied remote stress. This observation indicates that with more saturated multiple cracking, the fibers are more efficient in sharing the applied loading (so the matrix carries less) and this is one of the advantages of ECC members.

This model developed in this work provides a framework to predict the macroscopic mechanical behavior of ECC from parameters at the microscale. As there are many factors that can influence the ductility and durability performance (dependent on crack opening and crack number) of ECC, this model can be utilized to evaluate how these factors work, and facilitate the optimal design of ECC for various applications.

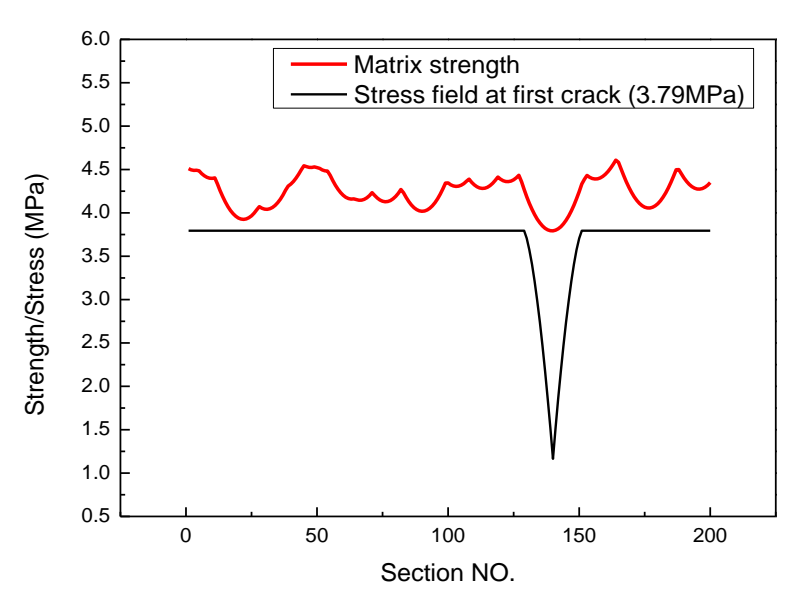

(a)

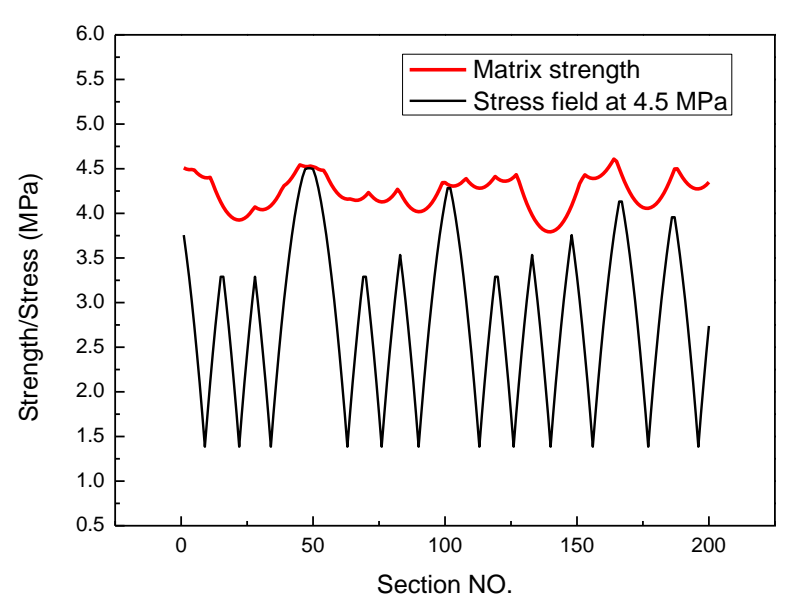

(b)

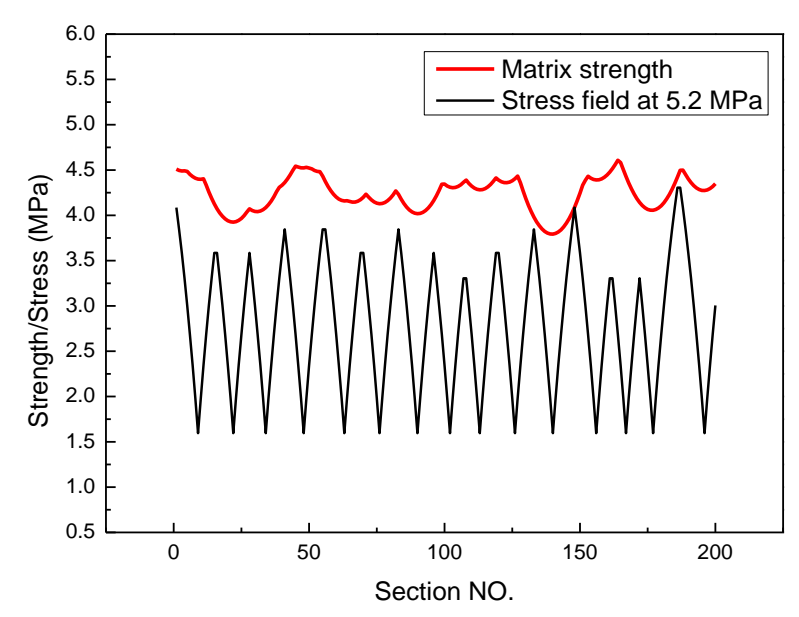

(c) 


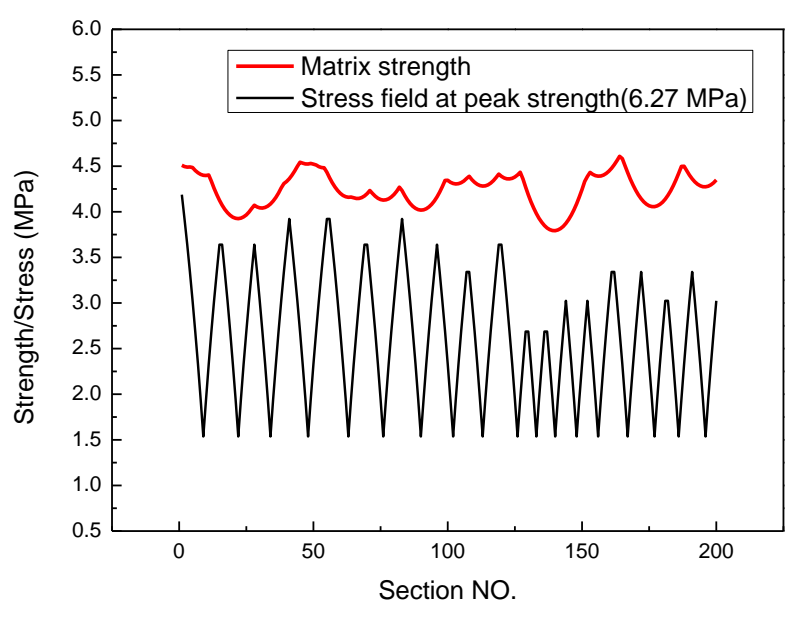

(d)

Figure 15: Stress field vs matrix stress at various stress level: a) first crack strength; b) 4.5 MPa; c) $5.2 \mathrm{MPa}$; d) peak strength

\section{CONCLUSIONS}

In this study, starting from the stress transfer of single fiber considering slip hardening, fiber rupture and other influential factors, the stress field near a crack is calculated. The matrix strength is distributed based on a stochastic approach which can reflect the correlation of strength between neighboring sections. By comparing the stress field with the matrix strength field at increasing remote tensile load, the formation and location of multiple cracks are determined. Stress vs strain behavior can then be derived. Simulated stress vs strain curves and crack number for a given ECC specimen was found to be in good agreement with test results. The model developed in this study should be applicable to the micromechanics-based design and optimization of ECC.

\section{ACKNOWLEDGEMENT}

The authors would like to thank Professor Ravi Ranade at University at Buffalo for providing the program of stress-crack opening relation which this paper refers to. Also, Financial support of this work by the Hong Kong Research Grant Council through GRF 615411 is gratefully acknowledged.

\section{REFERENCES}

[1] Li, V. C. 1993. From Micromechanics to Structural Engineering-the Design of Cementitous Composites for Civil Engineering Applications.

[2] Aveston, J., Mercer, R. A., and Sillwood, J. M. 1974. Fiber Reinforced Cements-Scientific Foundations for Specifications. in Guildford, Surrey, U. K., National Physical Laboratory IPC Science and Technology Press Ltd. Composites-Standards, Testing and Design pp. 93-103.

[3] Li, V. C., and Leung, C. K. Y. 1992. Steadystate and multiple cracking of short random fiber composites. Journal of Engineering Mechanics. 118(11):2246-64.

[4] Lin, Z., and Li, V. C. 1997. Crack bridging in fiber reinforced cementitious composites with slip-hardening interfaces. Journal of the Mechanics and Physics of Solids. 45(5):763-87.

[5] Yang, E., Wang, S., Yang, Y., and Li, V. C. 2008. Fiber-bridging constitutive law of engineered cementitious composites. Journal of advanced concrete technology. 6(1):181-93.

[6] Maalej, M., Li, V. C., and Hashida, T. 1995. Effect of fiber rupture on tensile properties of short fiber composites. Journal of engineering mechanics. 121(8):903-13.

[7] Kanda, T., Li, V. C. 1999. Effect of fiber strength and fiber-matrix interface on crack bridging in cement composites. Journal of Engineering mechanics. 125(3):290-9.

[8] Leung, C. K. Y. 1996. Design criteria for pseudoductile fiber-reinforced composites. Journal of engineering mechanics. 122(1):108.

[9] Wu, H. C., and Li, V. C. 1992. Snubbing and Bundling Effects on Multiple Crack Spacing of Discontinuous Random Fiber - Reinforced Brittle Matrix Composites. Journal of the American Ceramic Society. 75(12):3487-9.

[10] Kabele, P., and Stemberk, M. Stochastic model of multiple cracking process in fiber reinforced cementitious composites. 2005. Turin: CCI Centro Congressi Internazionale srl: Citeseer; Proceedings of the 11th international conference on fracture.

[11] Kabele, P. 2010 Stochastic finite element modeling of multiple cracking in fiber reinforced cementitious composites. Fracture and Damage of Advanced Fibre-reinforced Cement-based Materials. pp. 155-63. 
[12] Lu, C., Leung, C. K. Y. 2016. A new model for the cracking process and tensile ductility of Strain Hardening Cementitious Composites (SHCC). Journal of Cement and Concrete Research. 79:353-65.

[13] Wu, H. C., Li, V. C. 1995. Stochastic process of multiple cracking in discontinuous random fiber reinforced brittle matrix composites. International Journal of damage mechanics. 4(1):83-102.

[14] LU, C., YU, J., and LEUNG, C. K. Y. 2016 An Improved Image Processing Method for Assessing Multiple Cracking Development in Strain Hardening Cementitious Composites (SHCC), submitted to journal of Cement and Concrete Composites. 